\title{
DNA Chips: from elaboration to application
}

E. Souteyrand

École Centrale de Lyon, IFOS - UMR 5621 CNRS - 36 avenue de Collongue, 69139 Écully, France

\section{Introduction}

The Nucleic Acids (DNA and RNA) are molecules holding the genetic information in all life cells and constitute the crucial and fundamental component of genes monitoring the working of living organisms. The scientific projects actually put in place through the world indicate clearly the strategic importance of the complete gene knowledge of wide varieties of live materials (Human Genomic Sequencing, Genoplant, Bacteriology and Environment). In this way, a key point is the development of tools allowing the multiparametric analysis. Based on the molecular hybridization principle, DNA chips are considered as new and powerful tools combining the large integration ability of microelectronic devices with the properties of biological interaction for simultaneous analysis of several thousands nucleic acids. Compared with the microarrays on high-density membranes, the advantages of such devices hold in their miniaturisation allowing to collect an impressive number of information and the robotic process of the elaboration leading to a best reproducibility. Moreover, the very small size of each active area on DNA chips requires small amounts of biologic materials. Sequence analysis, genotyping and gene expression monitoring are some examples of potential applications in broad areas: biomedical research, genomics and clinical diagnostics, pharmacology, environment and food industry and give an idea of scientific and economic stakes. The importance of this tool for future gene studies leads to a strong competition between several teams which proposed different technological choices. Although issued from very simple concept, DNA Chip technology is complex because wide complementary competencies such as surface chemistry, micro-technology, electronics, molecular biology and data processing must be gathered.

Note that the required criteria for DNA chips will be determined from expected applications:

Low density: 10-1000 probes diagnostics

Medium density: 1000-10 000 probes mutations studies

High density: $10000-100000$ probes gene sequencing

Depending on the targeted application and the density of array, various technological ways can be looked to the DNA Chip implementation: for instance, the material support can be chosen to allow a direct immobilisation of synthesised oligonucleotides (electrochemical deposition required a conducting substrate) or to use an electronic detection of the hybridisation process (semiconductor substrate); and the appropriate surface chemistry will be different as a function of the employed substrate. One of challenges is to address precisely different products in defined areas at micrometer scale. For this, various processes are proposed and will be described later.

\section{Principle of DNA Chips}

Watson and Crick have described the nucleic acid structure as a double helix conformation of two polynucleotide strands. Each strand is constituted of polymeric chain bearing phosphate and sugar groups on which are attached the bases: Ademine, Thymine, Cytosine and Guanine. These bases are complementary by two through three hydrogen bonds in the C-G base pair and two in the T-A base pair. This base-pairing property gives the ability of one single strand to recognise his complementary strand to form a duplex (Fig. 1). So DNA sensors consist to immobilise onto a solid support well-defined sequences of single strands as biological receptor.

Thus, DNA chips associate this base pairing properties with the performances of the microtechnology. DNA chips are miniaturised and high density arrays of molecular probes (currently oligonucleotides) on which the targets (unknown DNA single strands to be detected) could be hybridised. Each type of known and well identified probes are located on the solid surface at a predefined position in the array. Then, device is put in contact with solution containing the targets to be detected. When probes and targets are complementary, the molecular hybridisation occurs on the solid support indicating the biochemical recognition. The detection of areas on the matrix where hybridisation is complete allows to identify and/or to quantify the single strand target (Fig. 2). Currently, the detection of hybridisation events is performed by using markers fixed on targets.

First experimental parallel analysis performed on glass slide evidenced several factors influencing the duplex formation such as the effect of oligonucleotide length, the base composition or the sequence complexity [1]. Besides this, the overall kinetics of DNA hybridisation is strongly influ- 


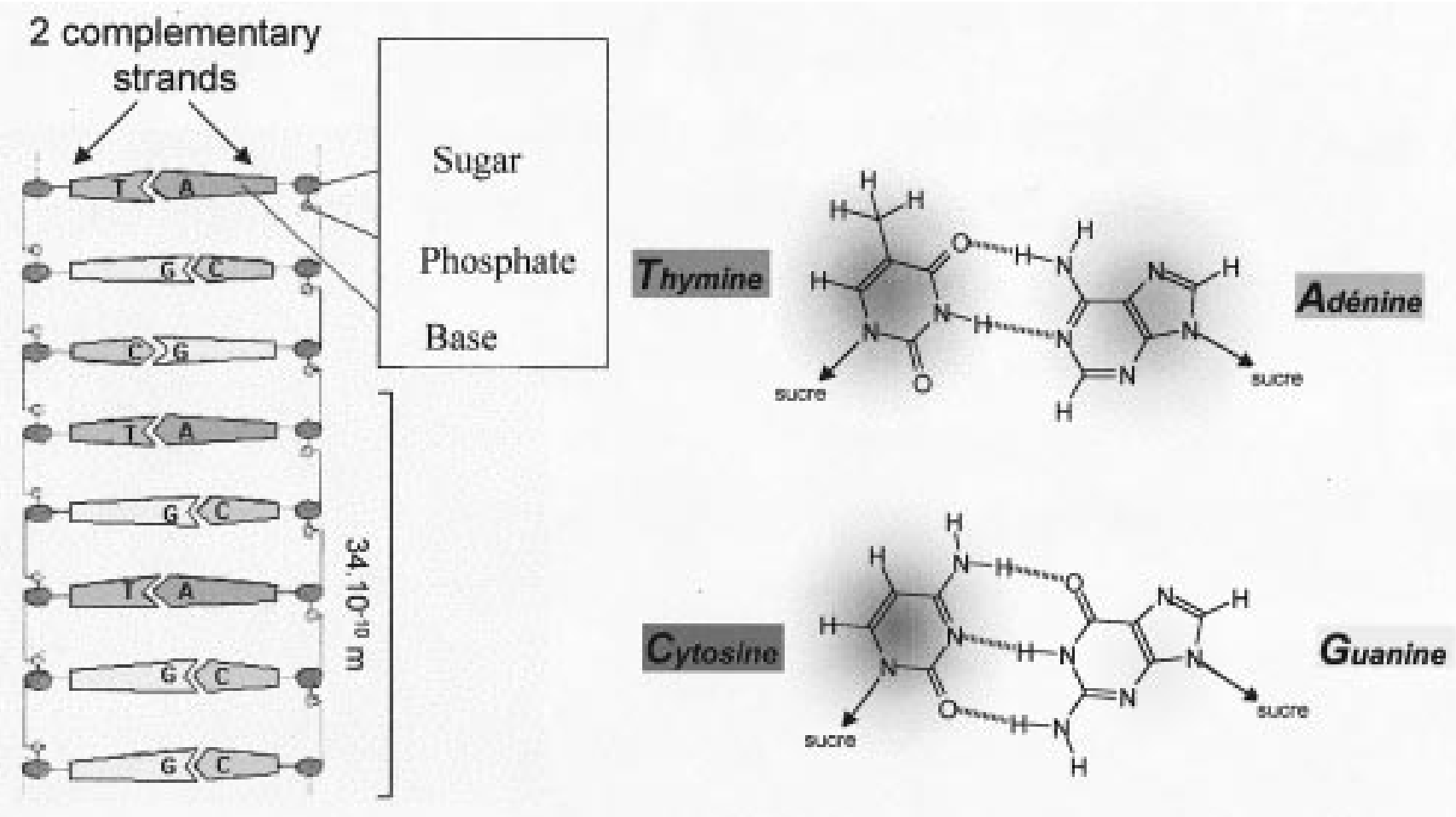

Figure 1. Schematic picture of DNA and principle of base pairing.

Double stranded DNA molecules are formed of two polynucleotides in double helix structure. Each polynucleotide is made of successive deoxyribonucleotide linked together. Each deoxyribonucleotide consists of the sugar linked to a phosphate group and one of four bases: Adenine, Thymine, Cytosine and Guanine.

Base pairing between the two strands involves the formation of hydrogen bonds between bases. The only permissible combination is $A$ base-paired with $T$ through two hydrogen bonds and $C$ - $G$ base pair with three hydrogen bonds. As a consequence, only two complementary strands can to be hybridised.

enced by the fact that DNA probes are immobilised on a solid support. From mathematical model based on receptorligand interactions, Chan et al. [2] determined that main parameters as target concentration in solution and surface probe densities must be optimised for improving hybridisation process on solid support. Thus, the steric overcrowding surrounding each DNA probe bound on the surface must be taken into account for access ability of targets and also to avoid quenching phenomena in the case of fluorescence labelled targets. One other point for hybridisation detection is to distinguish the real signal relative to hybridisation process from the signal connected to adsorbed targets between probes fixed on surface. The stringency of hybridisation solutions, their salt concentrations, the operate temperature, the washing solution composition and rinsing protocols play crucial role in the success of the hybridisation step and as consequence on the detected signal. [3].

Moreover, the use of spacer molecules to reduce steric interference from the support on the hybridisation behaviour of immobilised probes has been investigated [4-5]. The optimal spacer length onto an amine functionalised polypropy- lene support was determined to be at least 40 atoms length giving up to 150 fold increase in the yield of hybridisation. But, we must keep in mind that both negatively and positively charged groups in the spacer can diminish this yield.

\section{Different steps of DNA implementation}

The main steps in the elaboration of DNA chips concern:

1- the choice of solid support

2- Surface preparation

3- Immobilisation of presynthesized oligonucleotides or direct synthesis on the support

4- Addressing of reagents

5- Detection of hybridised areas

6- Data processing

Note that all these steps are not independent each from other and can be imposed by the chosen technology way. 


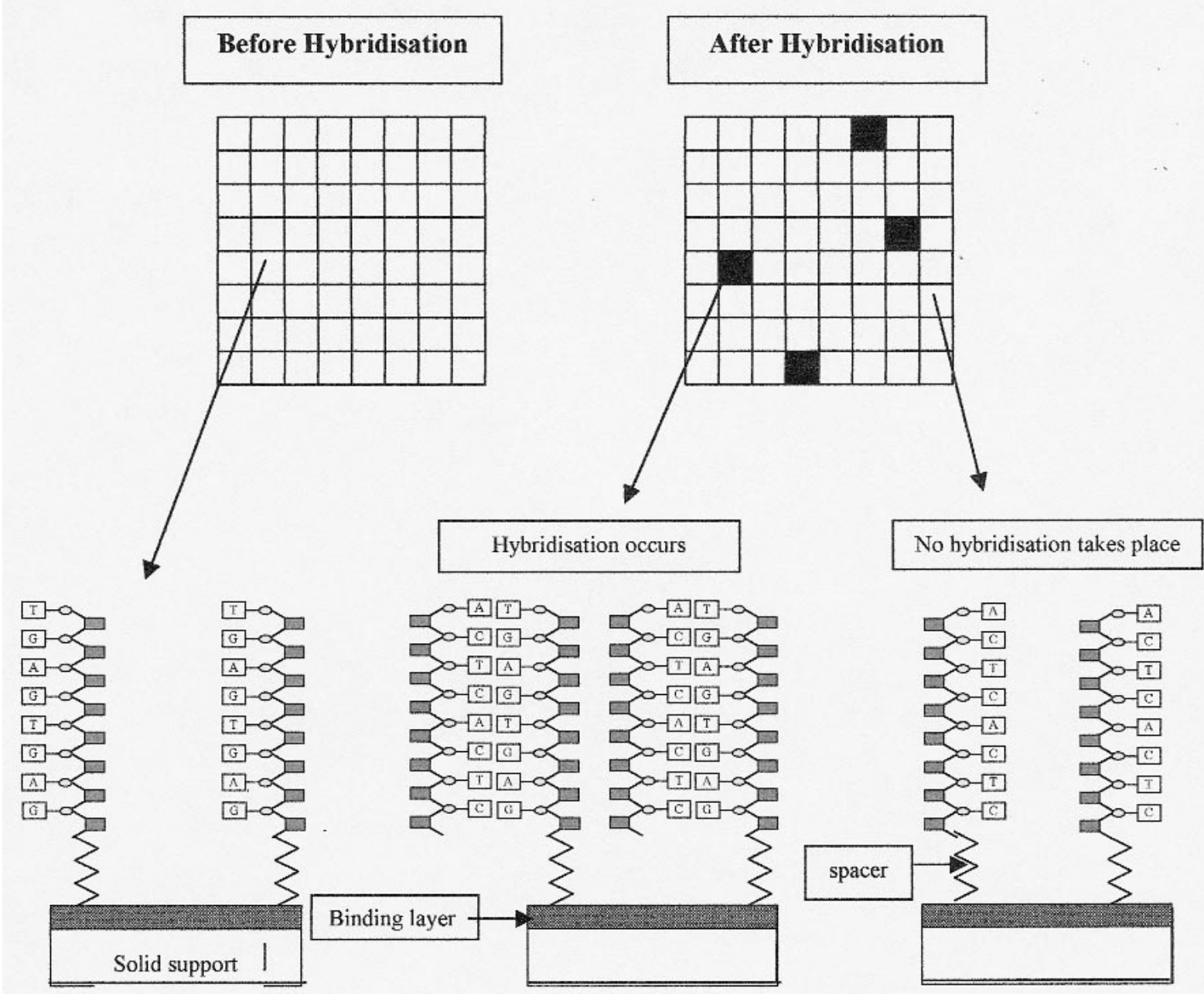

Figure 2. Principle of DNA chips.

On a solid support, a lot of defined areas are drawn. In each area, known oligonucleotides are immobilised (or directly synthesised). Each area corresponds to one type of oligonucleotide. When DNA chips is put in contact with solution containing single strand DNA to be detected, only strands complementary to fixed oligonucleotides can form a stable hybrid in localised area. The detection of different areas where hybridisation occurred allowed determining the various sequences present in solution.

\section{Choice of solid support}

One of the most used materials is the glass slide because of low cost and its transparency. Metallic substrates are also employed for conductive properties (Au or Pt electrodes). In fact, metallic dots corresponding to active areas are supported on silicon substrate inside conducting lines are drawing for individual electronic addressing. Structures made of silicon covered with thermal silicon dioxide layer are also used for direct detection of hybridisation trough the field effect.

\section{Surface preparation}

Whatever the chosen solid support, a surface preparation is required in order to attach robustly the probes on the sur- face. This step aims to create reactive sites on which either the fixation of oligonucleotides or the start of DNA synthesis is possible. Generally, a cleaning procedure (hydroxylation step) performed on silica surface of glass slide or $\mathrm{Si} / \mathrm{SiO} 2$ substrate leads to the formation of hydroxyl groups on the surface. A direct coupling between DNA strands (with or without linker) and $\mathrm{OH}$ terminal groups are strongly unsuitable because the Si-O-C binding is easily cleaved. A silanisation process is expected to improve the immobilisation of probes on surface. The silane must bear two different terminal groups: one extremity of mono,di or tri functional $\mathrm{Si}$ atoms able to bind with the surface hydroxyl groups whereas the other end must be an hydroxyl, amine or carboxyl acid function in view to graft DNA. 
The formation of a monolayer is expected by using a mono-functional silane constituted of one reactive group to react with silanol silica surface groups. Nevertheless the weak reactivity with the surface due to the presence of only one terminal group must be noted. By contrast, the use of di- or tri- functional silane would increase the reaction yield; in fact the terminal groups can react either with the surface silanols or in bulk with adjacent hydrolysable groups of silane and finally give birth to a 3 dimensional and bad structured layer.

This silanisation step is extremely important and determining for future performances of the chips. An unsuccessful functionalisation process would induce a lake of reproducibility and an unstability of device. A lot of publications indicate the difficulties to control the silanisation reaction strongly depending on the experimental conditions.

Various type of silane have been used:

On glass substrates, Pease et al. [6] proposed the following chemical treatment: after cleaning in concentrated $\mathrm{NaOH}$ and rinsing in water, surfaces were derivatized for 2 hours with a solution of $10 \%$ (vol./vol.) bis (2-hydroxyethyl)aminopropyltriethoxysilane in $95 \%$ ethanol and ether, dried in vacuum at $40^{\circ} \mathrm{C}$ and heated at $100^{\circ} \mathrm{C}$ for 15 minutes. Then a linker is attached by reacting substrates with 4,4 ' dimethoxytritil (DMT)-hexaethyloxy-O-cyanoethyl phosphoramidite.

Southern's group proposed the deposition of 3Glycidoxypropyltrimethoxysilane (GPTS) on glass surface (in 1992) [7] and more recently on $\mathrm{Si} / \mathrm{SiO}$ substrates (since 1997) [8] before starting a direct synthesis of oligonucleotide on solid support . Lamture and al [9] used the same silane for immobilising presynthesised oligonucleotides. In the first case, the epoxide residues were cleaved in acid solution to yield a primary hydroxyl group required for next synthesis. In the second case, reaction performed by adding 3' amine modified oligonucleotide probes in $0.1 \mathrm{M} \mathrm{KOH}$ at $37^{\circ} \mathrm{C}$ for 6 hours in air regulated in $100 \%$ relative humidity leads to a covalent attachment of ODN on surface. (Fig. 3).

In the two cases, a stable and covalent binding is expected but the robust attachment of biomolecules on support is function of various operate conditions during the silanisation process and the next chemical treatment : concentration of silane, solvant, water amount, temperature, reaction time, the use of catalyst, the choice of linker are some factors to be taken into account. The stability in time and the compatibility of this underlying layer with successive chemical treatments are determining criteria for making reusable devices. A detailed review of publications about DNA chips does not give clear evidence of the covalent binding and it seems that in the most of cases the oligonucleotides were just adsorbed on surface. Although a large part of oligonucleotides was removed under washings, they remain hybridisable.
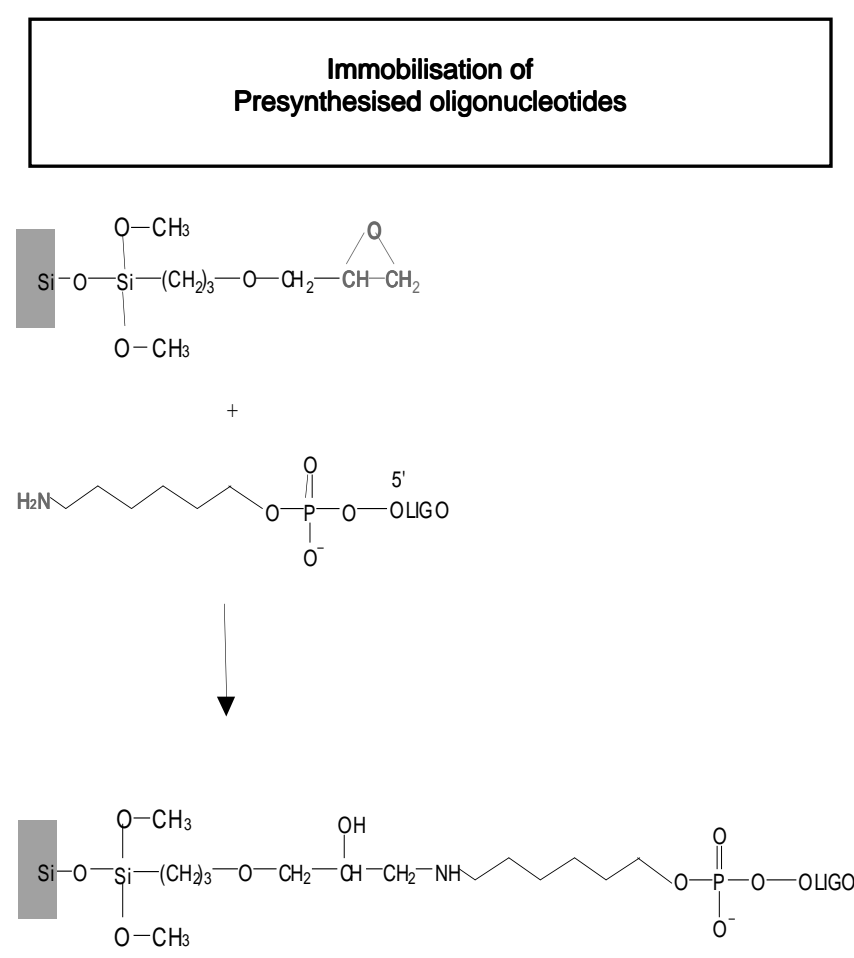

Figure 3. Immobilisation of presynthesised oligonucleotides onto solid surface.

In $\mathrm{KOH}$ solution, aminolinker fixed to 5 ' end oligonucleotide reacts with terminal epoxide group of GPTS to form a covalent binding.

\section{DNA Synthesis cycle}

Currently, the direct synthesis of oligonucleotides on solid support is made by using the phosphoramidite chemistry .

A synthesis cycle of oligonucleotides on solid support includes 6 steps whose 4 are repetitive steps (Fig. 4):

- Fixation: the 3' OH from terminal nucleoside is attached to substrate via an ester or succinate function.

1- Deprotection: DMT protecting group is removed by Trichloroacetique acid (TCA) to active the nucleoside for next coupling.

2- Coupling: addition of 3 ' activated phosphoramidite for reacting on 5 ' end fixed nucleotide.

3- Capping: this step consists with acetylation process to hinder the unreactive nucleotides of this step to act in following cycles.

4- Oxidation: the internucleotide phosphodiester bond with trivalent Phosphor is chemically unstable and must be transformed in pentavalent form by using a mixture of iode, pyridine and water. 


\section{Direct Synthesis of oligonucleotides}

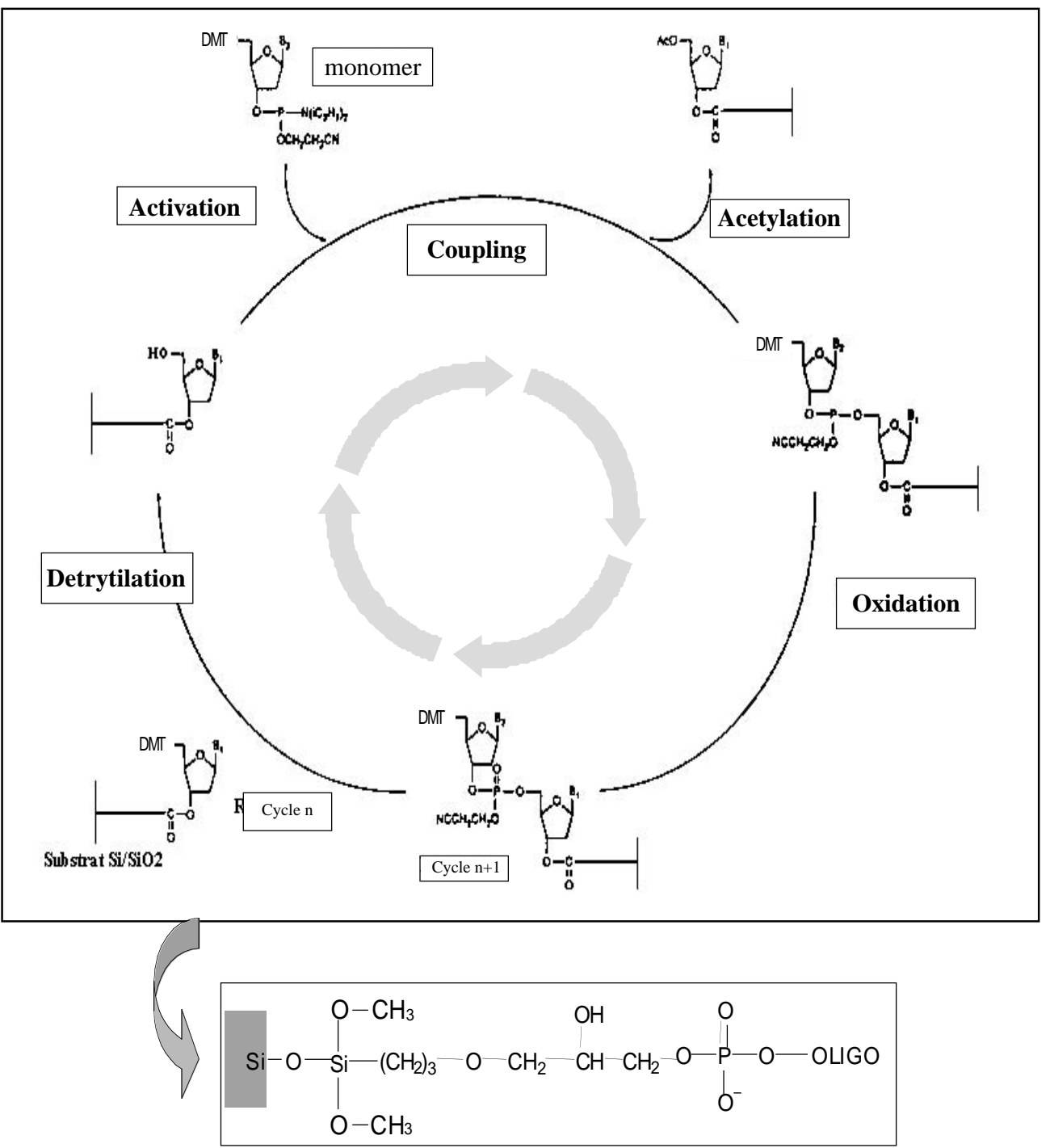

Figure 4. Cycle of oligonucleotide synthesis.

$\mathbf{N}$ cycle correspond to the formation of $\mathbf{N}$ mer length oligonucleotide.

- A total deprotection with $\mathrm{NH}_{4} \mathrm{OH}$ gets off all protecting groups on heterocyclic bases and yields free the $5^{\prime}$ and 3' $\mathrm{OH}$ terminals.

\section{Addressing of reagents}

Once the substrate chemically prepared for next step, the problem is to address reagents in confined areas. Reagents can be purified presynthesised oligonucleotides for immobilisation or all reagents necessary for direct DNA synthesis on microsurfaces.
Immobilisation of presynthesised oligonucleotides is well adapted for low or medium density array but cannot be realist for high-density array. (That would mean the preparation of more 100000 solutions containing each type of oligonucleotides). Direct synthesis of oligonucleotides is a strategy opening a way to a high-density array. But, one problem concerns the non-control of the possible failure during the synthesis.

Different working groups have proposed various addressing techniques. 


\section{Mechanical addressing}

As far as 1989, Mirzabekov et al. [10-11] proposed a technology of sequencing by hybridisation to oligonucleotide microchips (SHOM) using a matrix of gel elements fixed on glass plate. The polyacrylamide gel was activated by substitution of some amide groups with hydrazide groups by hydrazine-hydrate treatment. The presynthesised oligonucleotides were transferred from solution on the micromatrix element thanks a pin-robot. Specially, the pin temperature was kept at a given point to prevent drop evaporation or water condensation on the pin. From the authors, 0.550 fmol of oligonucleotides can be immobilised per microelement of the size 40x40x20 $\mu \mathrm{m}$. Advantages of this technology are connected with its simplicity and low cost. The 3D structure of gel element allows both a sufficient immobilisation of oligonucleotides without any drastic precaution and facilitates the hybridisation process.

\section{Electrochemical deposition of oligonucleotide via pyrrole groups}

Presynthesised oligonucleotides (ODN) were previously linked covalently to a pyrrole. Then, the electrochemical copolymerisation of pyrrole mixed with pyrrole-ODN gives in one step, an adherent conducting polymer layer on the surface. This method requires conductive surface ( $\mathrm{Pt}$ or Au have been used) and a selecting switching of the electrodes to polarise individually each element of the matrix. A perfect electrical insulation between each active areas is also necessary and limits the decrease in size of such DNA array [12].

\section{Electronic addressing}

Based on the strong negative charge of DNA beard by phosphate groups, metal active sites are designed on semiconductor substrate. Thanks a multiplexing device, the sites can be selectively polarised with a positive sign. As a consequence, the negatively charged probes present in a DNA solution are fastly driven to the positively charged sites and this high concentration of reagents on site improve the kinetics of chemical binding on this site.

Similar process allows the move and the concentration of targets molecules on test sites and the hybridisation rate is strongly accelerated. Unbound or non specific bound DNA from each site can be removed by simply reversing the polarity of site. This electronically activated system needs low concentrations of DNA molecules and reduces the time for hybridisation [13-14-15].

\section{Addressing via photolithographic process}

S. Fodor's group proposed to combine the photolithographic process with the direct synthesis of oligonucleotides on glass support. In this technique, the DMT protecting group used in classical chemical synthesis as described before is rem- placed by a photolabile protecting group. The use of photolithographic masks allows to define the specific areas on which light is directed and can remove the photolabile protecting groups. The opened areas are then active for first coupling with one type of nucleosides. Each nucleoside bears a photolabile protecting group at the 5' end (MeNPoc -dT; MeNPoc -dC; MeNPoc -dG ; MeNPoc -dA for instance) and will be available for next coupling only after deprotection under light. This technology combining photolithographic process with the phosphoramidite chemistry leads to a rapid made of high density DNA matrix. Using a limited set of masks, it is easy to obtain all the combinatorial synthesis of sequence on support from the four fundamental bases (A, T, C, G). For instance, the number of possible probes of 8 mers length from the four fundamental bases is $4^{8}=65536$ and requires only $4 \times 8=32$ chemical steps. This addressing is particularly well adapted for systematic read out of unknown gene sequences. (genomic sequencing). The only one drawbacks is relative to the photodeprotection yield in the range $85 \%-98 \%$ at each step which can limited the length of synthesised oligonucleotides [6-16-17-18].

Spatial resolution achievable with this method would be correspond to individual active area size of 5-10 $\mu \mathrm{m}$ with densities of $10^{6}$ sequences $/ \mathrm{cm}^{2}$. Unfortunately, the conventional projection lithographic process faced to diffraction limits decreasing the required high contrast $(1 / 100)$ between illuminated and masked areas. So successful studies aiming to put semiconductor photoresist in place of projected masks have been reported [19].

\section{Ink jet technology}

Another promising way is the use of ink jet printer heads to deliver microdrops $(100 \mathrm{pL})$ of reagents on surface. This technique allows either immobilisation of presynthesized ODN or a direct synthesis of DNA sequences without protecting groups. In this case, problem of the photolabile deprotection reaction yield is avoided. Nevertheless, the fast solvent evaporation required to make synthesis cycle under saturation conditions. Drops must be confined in small areas. To prevent drop spreading and reagent mixing, active areas can be defined thanks usual photolitographic process: substrate was overall covered with a photoresist film. A mask drawing 100000 circles of $100 \mu \mathrm{m}$ diameter with a $30 \mu \mathrm{m}$ distance between them is applied. Light directed through the mask removed the resin out of the circles. A vapour phase deposition of tridecafluorotetrahydrooctyl-triethoxysilane gives hydrophobic properties at this surface. Then, protecting resin on the circle surface is peeled off and chemical treatment is performed to provide a hydrophilic character on this active areas (Fig. 5) [20]. Photographic process is employed only at the first step to design the DNA chips. Parallel adressing on different located sites of reagent drops 


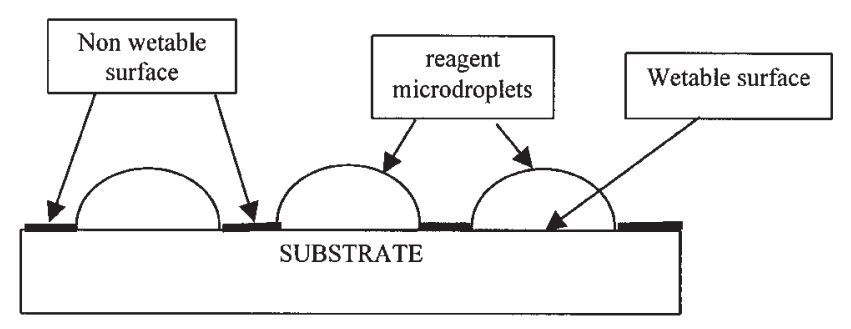

Figure 5. Drops are confined on the surface thanks a previous chemical surface treatment giving hydrophobic or hydrophilic properties on local areas.

ejected by piezzoelectric devices allows a large versatility to built simultaneously various oligonucleotides.

\section{Detection of hybridisation}

Usually the detection of hybridisation on DNA chips needs the labelled targets and takes from classical biological analysis methods as immunoassays, or dot blot techniques. An ideal label for DNA hybridisation detection on solid support must have following properties: to be easily attached to DNA target, to be detectable at very low concentration, to be stable in various hybridisation solutions, to be not perturbed by the solid support, or by the steric hindrance on the surface. Various substances can be used and have been extensively listed in J.A. Matthews and L.J. Kricka's review [21]. The potential labels can be classified as radioactive, fluorescence, enzymatic elements.

Radioisotope labels such as ${ }^{125} \mathrm{I},{ }^{32} \mathrm{P}$ or ${ }^{33} \mathrm{P}$ have proved their efficiency and their high sensitivity and the possibility to be quantitative method but their weak spatial resolution $(300 \mu \mathrm{m})$ prevents their use with high integrated arrays. Moreover, the associated problems of safety, stability, waste disposal and high cost have turned off studies towards alternative solutions.

Luminescent or fluorescent entities are also employed. For instance, Fluoresceine, Rhodamine, Cys3 and Cys5 are groups directly bounded to the DNA targets. A double labelling technique has also been developed: the DNA targets were just biotinylated; the small size of biotin molecule does not perturb the hybridisation process. After hybridisation, the formed duplexes were fluorescent labelled with streptavidin-phycoerythrin conjugate thanks the strong affinity between streptavidine and biotin [22]. Detection by fluorescent markers is sensitive with high spatial resolution, semi quantitative but requires to monitor the quenching and blenching phenomena.
The reading of hybridised areas via the detection of labels is performed by using several imaging techniques such as a scanning confocal fluorescence microscope for fluorecescent probes [6], a laser scanning associated with a Charge coupled device (CCD) camera [13] or a phosphorimager with radioactive elements.

A direct detection of hybridisation without the use of labelled probes is suggested by ECL group. Based on the negative charges present on DNA strands, the principle of the technique consists to follow the field effect induced by hybridisation process inside a blocking semiconductor structure [23]. Application of this detection technique to DNA chips, implies a number of electrical connections equivalent to the number of active areas. To avoid multiple electrical contacts, this group proposed to use the properties of semiconductor under light. The effect of charge induced by DNA hybridisation process can be detected through the change of surface potential by using a modulated light perturbation. Scanning the light on the surface, they can distinguish the hybridised areas without the use of labels [24].

\section{Applications of DNA chips}

The use of DNA chips of several hundred of thousands active sites containing all possible combinations of oligonucleotides will be particularly well adapted to the sequencing by hybridisation of unknown genes. Some problems relative to the repetition of sequences in the gene can lead to an indetermination. One solution is to increase the length of immobilised probes, that requires an improvement of DNA synthesis yield.

Another large field of interest of DNA chips concerns the simultaneous analysis of gene expression. That is why the first applications to prove the potentialities of DNA chips, aimed to determine quantitatively the expression level of 6200 yeast genes in Saccharomyces cerevisiae. [22]. 90\% of all yeast mRNA have been observed whose $50 \%$ were present at levels between 0.1 to 1 copy per cell. That gives the sensitivity scale. Using similar device, discrimination of one single nucleotide polymorphism. have been observed in HIV -1 clade protease gene [26].

M. Bellis and P. Casellas have reviewed the first applications made with the DNA chips [29] whereas B. Jordan analysed the possibilities of these new devices compared to conventional high density membranes [28]. All the authors emphasize the small quantity of required biological materials, the ability to detect very low expression level of RNA. As a conclusion they predict a light future for these devices. 


\section{Conclusion}

Challenge of the next century will be the understanding of all the information contained in genome, from the whole genome sequencing of all living organisms (human, vegetal, animal, bacteria,) to the gene expression analysis and the knowledge of the gene function. The extent of the work appears if we consider the bio-diversity of species and if we take in mind that only human genome concerns about 80000 genes and comprises approximately $3.10^{9}$ base pairs. The determination base per base of the entire genome can be a step but the main interest deals with the decoding of all the messages, the understanding of the functionality of genes or a set of genes and the mechanisms of gene interactions. In this context, DNA chips will be strictly necessary in molecular biology laboratories.

As we are at the birth of new technology, this small review evidences the multiple possibilities and wide technologic choices for the implementation of DNA chips. Futur advances in different fields (chemistry, nanotechnology, signal treatment, data processing...) would contribute to improve the performances of DNA chips both in term of miniaturisation specificity and sensitivity. The high activities in this field must be connected to the hope of scientists to have in hands this powerful tool.

\section{References}

1. Maskos, U.; Southern, E.M. Nucleic Acid Research 1992, 20, 1675-1678

2. Chan, V.; Graves, D.J.; Mc Kenzie, S.E. Biophysical Journal 1995, 69, 2243-2255.

3. Maskos, U.; Southern, E.M. Nucleic Acid Research 1993, 21, 4663-4669.

4. Shchepinov, M.S.; Case-Green; S.C.; Southern, E.M Nucleic Acid Research 1997, 25, 1155-1161.

5. Balladur, V.; Therez, A.; Mandrand, B. J. of Colloid and interface science 1997, 194, 408-418.

6. Caviani Pease, A.; Solas, D.: Sullivan, E.D.; Cronin, M. T.; Holmes, C. P.; Fodor, S. P. Natl. Acad. Sci. USA Biochemistry 1996, 91, 5022-5026.

7. Maskos, U.; Southern, E.M. Nucleic Acid Research 1992, 20, 1679-1684.
8. Gray, D.E.; Case-Green, S.C.; Fell, T.S.; Dobson, P.J.; Southern, E.M. Langmuir 1997, 13, 2833-2842.

9. Lamture, J.B.; Beattie, K.L.; Burke, B. E.; Eggers, M. D.; Ehrlich, D. J.; Fowler, R.; Hollis, M.A.; Kosicki, B.B.; Reich, R.K.; Smith, S.R.; Varma, R.S.; Hogan, M.E Nucleic acid research 1994, 22, 2121-2125.

10. Yershov, G.; Barsky, V.; Belgovkiy, A.; Kirillov, E.; Kreindlin, E.; Ivanov, I.; Parinov, S.; Guschin, D.; Drobishev, A.; Dubiley, S.; Mirzabekov, A Proc. Natl. Acad. Sci. USAGenetics 1996, 93, 4913-4918.

11. Mirzabekov, A. Biotechnology 1994, 12 , 27-32.

12. Livache, T.; Roget, A.; Dejean, E.; Barthet, C.; Bidan, G.; Teoule, R. Nucleic Acid Research, 1994, 22, 2915-2921.

13. Edman, C.F.; Raymond, D.E.; Wu, D.J.; TU, E.; Sosnowski, R.G.; Butler, W.F.; Nerenberg, M. N.; Heller M.J. Nucleic Acid Research, 1997, 25, 4907- 4914.

14. Sosnowski, R.G.; TU, E.; Butler, W.F.; O’Connell, J.P.; Heller, M.J. Natl. Acad. Sci. USA Biochemistry 1997, 14, 1119-1123.

15. Cheng, J.; Sheldon, E.L.; Wu, L.; Heller, M.J.; O'Connell, J.P. Anal. Chem. 1998, 70, 2321-2326.

16. Hacia, . J.G.; Brody, L.C.; Chee, M.S.; Fodor, S.P.; Collins, F.S. Nature Genetics 1996, 14, 441-446.

17. Chee, M.; Yang, R.; Hubbell, E.; Berno, A.; Huang, X. C.; Stern, D.; Winkler, J.; Lockhart, D.J.; Morris, M.S.; Fodor, S.P. Science 1996, 274, 610- 614.

18. Wallraff, G.; Labadie, J.; Brock, P.; Dipietro, R.; Nguyen, T.; Huynh, T.; Hinsberg, W.; McGall, G. Chemtech 1997, 22-32.

19. McGall, G.; Labadie, J.; Brock, P.; Wallraff, G.; Nguyen, T.; Hinsberg, W. Proc. Natl. Acad. Sci. USA - Applied Physical Sciences 1996, 93, 13555- 13560.

20. Blanchard, A.P.; Kaiser, R.J.; Hood, L.E.; 1996, 11, 687-690.

21. Matthews, J. A.; Kricka, L. J. Anal. Biochem. 1988, 169, 1-25.

22. Wodicka, L.; Dong, H.; Mittmann, M.; Ho, M. H.; Lockhart, D.J Nature Biotechnology 1997, 15, 1359- 1366.

23. Souteyrand, . E.; Cloarec, J.-P.; Martin, J.R.; Wilson, C.; Lawrence, I.; Michelsen, S.; Lawrence, M.F. J. Phys. Chem. 1997, 101, 2980-2985.

24. Souteyrand, E.;. Chen, C; Cloarec, J.P.; Nesme, X.; Simonet, P.; Navarro, I.; Martin, J.R. Applied Biotechnology and Biochemistry (March 1999).

25. Guo, Z.; Guiloyle, R.A.; Thiel, A. J.; Wang, R.; Smith, L. M. Nucleic Acids research, 1994, 22, 5456-5465.

26. Kozal, M.J.; Shah, N.; Shen, N.; Yang, R.; Fucini, R.; Merigan, T.; Richman, D.D.; Morris, D.; Hubbell, E.; Chee, M.; Gingeras, T.R. Nature Medicine 1996, 2, 753-759.

27. Schena, M.; Shalon, D.; Heller, R.; Chai, A.; Brown, P.; Davis, R.W. Proc. Natl. Acad. Sci. USA Biochemistry 1996, 93, 10614-10619.

28. Jordan, . B.R; J. Biochem. 1998, 124, 251-258.

29. Bellis, M.; Casellas, P. Médecine sciences 1997, 13, 13171324. 